\title{
La Universidad Católica Santa María La Antigua (USMA) a la vanguardia de los estudios de Psicología en Panamá
}

\section{Dra. Melva Palacios de Mon ${ }^{1,2, *}$}

${ }^{1}$ Directora del Programa de Doctorado en Psicología Clínica, Universidad Santa María la Antigua, Apartado Postal 0819-08550, Panamá, República de Panamá.

${ }^{2}$ Profesora titular de la Escuela de Psicología, Universidad Santa María la Antigua, Apartado Postal 0819-08550, Panamá, República de Panamá.

*Autor para correspondencia. Email: mpalacios@,usma.ac.pa

Recibido: 13 de marzo de 2014

Aceptado: 15 de abril de 2014

\section{Introducción}

Un ejemplar temático dedicado a la Psicología, de la Revista "Investigación y Pensamiento Crítico", nos plantea de inmediato el reto de hacer una reseña histórica y un análisis sobre lo que ha sido el desarrollo de la Psicología en la USMA, por dos razones: la primera de ellas, porque la USMA, con la creación de carreras y programas de Psicología, ha sido pionera en el país en todos los niveles académicos (Licenciatura, Maestría y Doctorado); y la segunda, porque al estar próxima a cumplir cincuenta años de haber sido fundada cabe destacar las distintas formas en que la Psicología ha contribuido al desarrollo, la consolidación y el prestigio de esta institución.

La universidad ha hecho énfasis, a través de sus programas de estudio en psicología, del compromiso que ha asumido con la comunidad. Este compromiso se logra gracias a la calidad de sus profesores, la dedicación y participación de sus estudiantes y su desempeño profesional en la comunidad a la que sirven. La proyección social de sus enfoques académicos capacita a los estudiantes para participar en proyectos comunitarios y analizado la condición humana mediante las tesis, los trabajos y los proyectos de investigación como podremos observar en el contenido de este resumen temático. 
Invest. pens. crit.

Vol. 2, No. 3, enero-abril 2014.

pp. 108-117

\section{Antecedentes históricos: creación de la primera escuela de psicología}

En los primeros años de la década de los sesenta cristalizaron las iniciativas para fundar una universidad católica y privada en la República de Panamá. Las diferentes gestiones que se habían hecho hasta entonces tomaron forma con la fundación de la Universidad Santa María La Antigua, que inició labores el 27 de mayo de 1965 y abrió sus puertas con ocho escuelas, entre las que se encontraba la Escuela de Psicología. Era la primera vez que se impartía la Carrera de Psicología en el país: algunos meses después, la Universidad de Panamá abrió también en el curso regular esta carrera.

La USMA desde su fundación en 1965 se hizo eco de la demanda que había en el país, por estructurar y ofrecer estudios de Psicología. Esta demanda también le fue presentada a la Universidad de Panamá especialmente por un grupo de profesores, la mayoría del área pedagógica, y por los estudiantes interesados en estudiar Psicología en Panamá.

La Escuela de Psicología formó parte de este proyecto universitario desde su gestación. Siempre hubo el interés y la intención de inaugurar la "nueva" Universidad con ofertas novedosas y, desde su creación y por su naturaleza, la USMA ha dado especial énfasis a las disciplinas que se vinculan con el ser humano. Al ser la Psicología una disciplina que tiene al ser humano como el eje central de su accionar, no es de extrañar que fuera una de las carreras que consideraran prioritarias por los fundadores de la Universidad.

Al consultar la obra "Historia Documentada de la Universidad Santa La Antigua" (Tomos I y II) de Bonifacio Difernan, profesor y ex-decano de la Facultad de Derecho y Ciencias Políticas de la USMA, podemos constatar lo ya señalado, cuando el autor nos transcribe algunos párrafos de las reuniones y Consejos iniciales, como el que citamos a continuación: "Se proyecta abrir en sus comienzos tres Facultades: Ciencias Exactas, Humanidades y Administración Pública y Comercio". Luego pasa a describir como estarán integradas las mismas y finalmente señala: "Además se abrirá un Instituto de Psicología para preparar personal en las siguientes especializaciones: Psicología Industrial, Psicometría, y Psicopedagogía y Orientación Vocacional y Profesional” (pp. 185)

En otros apartados de la citada obra, al explicar lo relativo a las Primeras Normas Académicas y en el Prospecto Informativo No. 1, aparece la Licenciatura en Psicología como una carrera que se imparte en la Escuela de Psicología; una de las siete escuelas que constituyen la Facultad de Filosofía, Letras y Educación (originalmente Humanidades). A continuación aparece una nota que señala lo siguiente:

Los estudios correspondientes a la Licenciatura y el Doctorado en Psicología con sus cuatro especializaciones: Psicología Clínica, Psicología Educativa (psicopedagogía), 
Invest. pens. crit.

Vol. 2, No. 3, enero-abril 2014.

pp. 108-117

Orientación Profesional y Psicología Industrial, se impartirán en un Instituto que es autónomo en lo docente, el cual, sin embargo, por razones de índole administrativa y de organización interna de la Universidad, al inicio de sus actividades dependerá de la Facultad de Filosofía y Letras. Esta autonomía es el paso previo para que se constituya en Facultad, en un plazo más o menos aproximado...

Y agregaba que

Tanto la estructura de los estudios de Psicología, en un organismo autónomo con características propias, definidas, así como la duración de los estudios y composición del curriculum, responden a las recomendaciones formuladas por los psicólogos en los últimos Congresos internacionales de la materia (pp. 308-309).

Lo anterior nos muestra cómo siempre hubo una consideración especial para la estructuración y desarrollo de esta Carrera, y cómo las autoridades universitarias de entonces reconocían la autonomía del campo de la Psicología y la necesidad de que pudiera constituirse en otra facultad más de la universidad. Casi 50 años después esta aspiración no se ha concretado. Quienes sí dieron el paso fueron las autoridades académicas de la Universidad de Panamá, que crearon en el año 2006 la Facultad de Psicología. Los estudios y la Carrera de Psicología de la USMA, han sido adscritos a diferentes Facultades a lo largo del tiempo, y desde el año de 1986 han formado parte de la Facultad de Ciencias Sociales.

En 1977 se extendió la formación de psicólogos al Centro universitario de la USMA en David, Chiriquí, siendo nuevamente la USMA pionera al impartir la Carrera de Psicología en el interior de la República, acción que incrementaría años más tarde con el ofrecimiento de algunas maestrías en este campo.

Los documentos históricos también nos muestran cómo desde sus inicios se reconocía la necesidad de una actualización en los estudios de Psicología, aspiración que se ha traducido en una revisión y ajustes periódicos de los planes de estudios de la licenciatura, incorporando en cada momento los últimas tendencias en la enseñanza de la Psicología y sus lineamientos pedagógicos; por ejemplo, de formación en competencias, los talleres desarrollo personal, las consejerías académicas, prácticas en todos los niveles de estudio y otras actividades complementarias no-cognoscitivas que garantizan un curriculum integral y actualizado. Esta misma tendencia de una actualización y desarrollo permanentes es la que ha impulsado a la creación de programas de formación avanzada en Psicología como son los programas de Maestrías y Doctorado a los que nos referiremos más adelante.

Los pocos profesionales de esta carrera que ejercían en Panamá antes de la fundación de la escuela en 1965, habían estudiado en el exterior, especialmente en países como Argentina, Chile y México, y algunos en Estados Unidos, países que ya tenían un amplio y reconocido recorrido académico y profesional en el campo de la Psicología. 
Invest. pens. crit.

Vol. 2, No. 3, enero-abril 2014.

pp. 108-117

\section{El campo profesional de la psicología}

Si bien la Psicología tiene un largo pasado histórico durante el cual se nutrió de la sabiduría, especialmente de la filosofía, tiene así mismo un corto tiempo como disciplina o ciencia autónoma que se estudia separadamente de la Filosofía, de la Medicina, de la Educación y de otros campos del saber. Como disciplina de estudio independiente se fundó en Europa a finales del siglo XIX. La Historia de la Psicología registra como un hito histórico la fundación en 1879, por el Psicólogo Wilhelm Wundt, del primer laboratorio de psicología en la Universidad de Leipzig en Alemania, fecha que la Psicología reconoce como su nacimiento como ciencia.

Es también importante destacar que la Psicología tiene varias ramas o campos de especialización y que el psicólogo en algún momento de su formación o de desempeño profesional se decanta por alguna de las siguientes especializaciones o énfasis:

\section{Psicología clínica:}

Podríamos decir que es el campo más explorado y también el más conocido por personas ajenas a esta profesión. Comprende el uso y la aplicación de principios teóricos y conceptos psicológicos para el diagnóstico y tratamiento de los problemas emocionales y de conducta. Por ejemplo, enfermedades mentales, estrés post-traumático, problemas de adaptación, familias disfuncionales y no-tradicionales, trastornos derivados de las adicciones, trastornos de la alimentación, conductas sociopáticas, etc.

La mayoría de los psicólogos clínicos ejercen en hospitales, clínicas e instituciones de atención específica tales como centros de rehabilitación, y en la consulta clínica privada. Trabajan en estrecha colaboración con profesionales de la medicina, como psiquiatras, pediatras, y neurólogos, así como también con trabajadores sociales, jueces o con la familia de los individuos que se atiende.

\section{Psicología educativa:}

Comprende tanto lo que se conoce como Psicología de la educación, como los aspectos que surgen de la relación maestro-alumno, rendimiento escolar y el propio proceso de aprendizaje. De allí que se designe con otros nombres y según el área que se trabaje, se denominaría Psicología educacional o Psicología escolar, y/o también Psicología del aprendizaje.

Algunos autores opinan que la que se designa como Psicología escolar, podría calificarse como psicología educacional aplicada. Todos estos psicólogos, trabajan en estrecha colaboración con profesores y maestros, con el alumno mismo y sus familiares, así como con las autoridades educativas en las áreas de planificación, curriculum y administración de la educación. 
Invest. pens. crit.

Vol. 2, No. 3, enero-abril 2014.

pp. 108-117

Cuando se trabaja con el estudiante, se tratan desde las dificultades de rendimiento y aprovechamiento escolar, los métodos de estudio, las estrategias para atender a niños excepcionales, los problemas de aprendizaje, y también incluye el área de la consejería y orientación vocacional y profesional.

\section{Psicología social y comunitaria:}

El psicólogo social se interesa en el estudio del comportamiento de los individuos en los grupos dentro de su contexto social, así como el efecto de los otros y de la cultura en la conducta individual. En este campo el psicólogo trabaja junto con sociólogos, antropólogos, trabajadores sociales, planificadores del desarrollo social y trabajadores comunitarios. Se tratan problemas relacionados con las actitudes, el cambio de éstas, los problemas de adaptación en los inmigrantes, las conductas que revelan prejuicios raciales o religiosos, las actitudes discriminatorias etc. Se desarrollan trabajos de investigación y programas comunitarios y de atención a grupos específicos.

Esta rama de la Psicología también se ocupa de las conductas antisociales, de los recluidos en cárceles y otro tipo de instituciones sociales, así como de las manifestaciones psicológicas que se observan en la participación ciudadana en los movimientos políticos, y del fenómeno del liderazgo y sus características.

Psicología industrial y organizacional:

Esta rama de la Psicología es algo más reciente que las mencionadas anteriormente, pero ha tomado un gran auge dadas las condiciones del desarrollo socio-económico y laboral de la sociedad contemporánea. En sus inicios y como consecuencia de la revolución industrial los psicólogos estudiaban la adaptación del hombre a la maquina: lo que hoy podría desplazarse a los fenómenos psicológicos derivados de la adaptación y uso de la tecnología como consecuencia del desarrollo de la informática especialmente en los últimos treinta años.

Este campo de la Psicología guarda estrecha relación con lo que se denomina Psicología Laboral o del Trabajo porque también se refiere al conocimiento de la estructura organizacional de las empresas y las relaciones laborales dentro de un organización, la capacitación en el puesto de trabajo, las características del reclutamiento de personal que dan lugar a una subespecializacion en Recursos Humanos.

Otras tareas propias de esta rama de la Psicología, se refieren a las asesorías a las empresas en material de desarrollo organizacional, la evaluación de las actitudes de los consumidores hacia los productos o servicios, el análisis de estrategias publicitarias, así como al diseño de programas de incentivo y bienestar en el trabajo. A algunas de estas actividades realizadas originalmente por Psicólogos, se les cataloga hoy día bajo el término de Consejería o "coaching”. 
Invest. pens. crit.

Vol. 2, No. 3, enero-abril 2014.

pp. 108-117

Los "psicólogos industriales", como generalmente se les llama, trabajan muy de cerca con los encargados de los departamentos de personal, administradores de empresas, directivos y creativos de la publicidad, jefes de comunicación institucional y gerentes.

Son estas cuatro las áreas más conocidas y consolidadas de la Psicología contemporánea, lo que se refleja también en los programas de postgrado que se ofrecen en las Universidades. No obstante, también debemos mencionar otras áreas, como por ejemplo, la psicología ambiental, que formaría parte de la perspectiva ecológica contemporánea. La psicología evolutiva que se centra en la investigación del desarrollo de los seres humanos, haciendo énfasis en los aspectos psicológicos propios de cada periodo; a saber, la infancia, la adolescencia, la edad adulta y la vejez. También la psicología del deporte, que involucra a los psicólogos en un trabajo especializado con los deportistas y los equipos deportivos.

Luego de este breve panorama de las áreas de la Psicología, podríamos afirmar que ahí donde se encuentre el ser humano desplegando cualquier actividad, seguramente la Psicología siempre tendrá algo que decir. Lo anterior podría resumirse en las palabras del psicólogo estadounidense J. Whittaker (1984):

“... no basta la tecnología para resolver los problemas sociales del hombre. Es el ser humano, y más exactamente su comportamiento, lo que obstaculiza la solución de la mayor parte de los problemas humanos."

\section{El ejercicio profesional y la reglamentación de la psicología en Panamá}

Dada la amplitud del campo profesional de la psicología, los primeros profesionales llegados a Panamá, antes de la creación de las Escuelas de Psicología, de la USMA y de la Universidad de Panamá, vieron la necesidad de organizarse gremialmente y es así como, también el 22 de junio de 1965, se funda la Asociación Panameña de Psicólogos (APP).

Esta Asociación, junto con las actividades desarrolladas por estudiantes y profesores en las dos Escuelas de Psicología, permitió la consolidación del quehacer psicológico en el país, proyectando en la comunidad la importancia y pertinencia de la formación de los profesionales de la Psicología.

Años después por gestión de los miembros de la APP se aprobó la Ley que reglamenta el ejercicio profesional de la psicología en Panamá y como consecuencia de ésta se crea el Consejo Técnico de Psicología (CTP), que emite la Certificación de idoneidad profesional (CIP) para el ejercicio de la Psicología en sus diferentes especializaciones. 
Invest. pens. crit.

Vol. 2, No. 3, enero-abril 2014.

pp. 108-117

\section{Creación de la primera Maestría en Psicología}

En 1990 la Escuela de Psicología celebró sus Bodas de Plata e inició las gestiones para ofrecer estudios a nivel de Maestría. El interés de algunos egresados de la escuela y la coyuntura nacional de la puesta en marcha del Código de la Familia, facilitaron que la Universidad ofreciera a partir del año 1992 la primera Maestría en el campo de la Psicología con el Programa de Maestría en Ciencias de la Familia con dos énfasis, a saber: la Especialización en Terapia Familiar, dirigida a profesionales de la salud mental, psicólogos y psiquiatras; y la Especialización en Orientación Familiar dirigida a otros profesionales relacionados con estos temas, como son los trabajadores sociales, abogados y jueces, enfermeras, consejeros vocacionales, pedagogos, sacerdotes y pastores.

Los estudios en Ciencias de la Familia exponen a los participantes en esos programas a distintos modelos de terapia familiar, tanto en la teoría como en la práctica, mediante la supervisión de las prácticas profesionales.

Desde sus inicios la USMA asumió un sólido compromiso con el estudio interdisciplinario de los temas relacionados con el matrimonio, la pareja y la familia. Como consecuencia de lo anterior, creó también en 1992 la primera Clínica Psicológica y de 'Terapia Familiar (CPTF), que ha dado servicio por más de 20 años a estudiantes, profesores y administrativos de la propia Universidad, así como al público que refieren las ONG y las instituciones gubernamentales de atención en salud mental a la población. La CPTF, desde su creación, ha sido un espacio de aprendizaje para los que estudian Psicología en la USMA en los distintos programas y niveles. También fue pionera esta Universidad en habilitar dos áreas con Cámaras de Gessel, recurso que permite un aprendizaje supervisado de las estrategias clínicas aplicadas y que constituyó, en su momento, una innovación y continua hoy día prestando el apoyo requerido.

\section{La creación de nuevas maestrías en Psicología y programas de especialización}

Esta visión pionera y de compromiso de la USMA en lo que a la Psicología se refiere, quedó demostrada una vez más con el ofrecimiento de otras maestrías. Así en el año 2005 se inició la Maestría en Psicología Industrial y Organizacional, para atender las necesidades de las organizaciones, particularmente del sector privado, con una formación sólida en los requerimientos del mundo empresarial y también del sector gubernamental y sus instituciones. La economía de servicio, propia de un país como Panamá y el crecimiento económico que se registra especialmente en los últimos anos, sustentan la pertinencia y urgencia de profesionales capacitados en la comprensión de la psicología de las organizaciones.

Dos años más tarde, en el 2007, se crea la Maestría en Psicología Clínica de Niños y Adolescentes para responder a la demanda de los profesionales de la Psicología que estaban interesados en contar con estudios de especialización para la atención de estos segmentos de la población. Esta 
Invest. pens. crit.

Vol. 2, No. 3, enero-abril 2014.

pp. 108-117

Maestría también se ofrece en la Sede de la USMA de David, Chiriquí, contribuyendo a elevar el perfil profesional de los psicólogos del área que trabajan con la población infantil y adolescente del interior de la República. Este mismo programa se ha ofrecido en dos oportunidades en la Sede de la USMA en Azuero.

Con el Patrocinio de la Organización de Estados Americanos (OEA), a través del Instituto Interamericano del Nino con sede en Uruguay, se dictó en una sola oportunidad y con una modalidad virtual, la Maestría en Políticas Publicas de Niñez y Adolescencia. Este programa de carácter internacional estaba orientado a capacitar planificadores y operadores de programas vinculados a instituciones públicas y privadas de protección a la infancia. En la actualidad se adelantan gestiones para retomar esta iniciativa y actualizar el plan de estudios para volver a incluirla en la oferta académica de la Universidad.

Por otro lado, con la colaboración del Instituto de Salud Mental del Ministerio de Salud (INSAM), en el año 2001, se elaboró el plan de estudios para ofrecer una Maestría en Prevención y Tratamiento de Adicciones. A la fecha no se ha ofrecido como maestría; en su lugar, se han organizado varios grupos que han tomado un Diplomado en esta misma temática. Anteriormente, en la década de los ochenta, se tenía un Convenio con la Cruz Blanca y se dictaban en la USMA seminarios y cursos de especialización en el tratamiento de adicciones, en colaboración con la Universidad de Gainesville de la Florida.

También en una sola ocasión, se ofreció un Diplomado a nivel de postgrado en "Teorías Psicoanalíticas", y más recientemente, en el año 2012, se dictó un Diplomado en "Psicología Analítica Jungiana”, con la colaboración de la Sociedad Venezolana de Analistas Junguianos (SVAJ) y el Centro de Estudios Junguianos de Caracas, Venezuela.

\section{Primer Doctorado en Psicología en Panamá}

Iniciado el nuevo siglo XXI, y después de casi cuarenta años de formar profesionales de la Psicología en la USMA, el paso siguiente parecía ser lógicamente la creación de un programa al más alto nivel: el Doctorado. Fue así como a partir del año 2001 se iniciaron las gestiones para estructurar el curriculum del que sería el primer Doctorado en Psicología que se ofrecía en Panamá. Con una Lección Inaugural se dio inicio en el segundo semestre del año 2003 a este programa, cuya primera promoción estuvo integrada por 22 candidatos. Una vez más, la USMA a la vanguardia en la enseñanza y el desarrollo de la Psicología en Panamá.

El Doctorado que se propuso fue un Doctorado en Psicología Clínica, al ser esta el área de la Psicología que tenía mayor demanda e interés por parte de los psicólogos, pues un gran número de ellos labora en el sector salud y otros en la práctica privada en Psicología Clínica. 
Invest. pens. crit.

Vol. 2, No. 3, enero-abril 2014.

pp. 108-117

Se concibió el programa con un alto componente internacional en su cuerpo docente, dado que así se le daría una mayor proyección en el exterior y se enriquecería la formación de los estudiantes con la experiencia de profesionales extranjeros de diferentes escuelas de formación y con amplia experiencia. Como requiere toda formación a un nivel doctoral, se escogieron varias líneas de investigación dentro de las cuales los candidatos podrían eventualmente encauzar sus trabajos delas diferentes materias y finalmente sus tesis.

Luego de 11 años de ofrecer el Doctorado en Psicología Clínica se han formado cuatro promociones y un número significativo han sustentado sus tesis de grado, lo que con toda seguridad ha contribuido a elevar el nivel de la práctica psicológica en el país y ofreciendo una atención de gran calidad a los que solicitan los servicios de un psicólogo.

\section{Consideraciones finales.}

$\mathrm{Al}$ analizar con mayor detenimiento la variedad de ofertas innovadoras y el rigor académico de las mismas, que en el campo de la Psicología se han ofrecido en estos casi cincuenta años que tiene de fundada la Universidad, no es exagerado decir que la USMA siempre ha estado a la vanguardia en la enseñanza y desarrollo de la Psicología en nuestro país, no solo por los programas que ha desarrollado, sino también por las actividades extracurriculares que ha estimulado y apoyado.

Los profesores y estudiantes de Psicología han participado de congresos nacionales e internacionales y la Universidad ha sido sede de muchos de ellos. Igualmente, ha acogido múltiples talleres y seminarios; se han dictado conferencias de alto nivel con conferencistas de distintas universidades, países y especialidades, lo que ha contribuido a elevar el prestigio bien ganado, que reconocen propios y extraños, en lo que a la enseñanza de la Psicología ofrece la Universidad Católica Santa María La Antigua.

El reto que tenemos, en adelante, es mantener la calidad de lo logrado y encauzar, con la misma dedicación y seriedad académica, las actividades de investigación y la publicación de los trabajos desarrollados en ese ámbito. Para lograr esta meta habría que considerar sus exigencias en la planeación estratégica de la Universidad, así como también se requiere de la vinculación con organismos que favorezcan dichas actividades, y la capacitación de los que se desempeñan en el área de la Psicología.

En el pasado se han publicado, en la Revista La Antigua, al menos dos números temáticos en Psicología, con trabajos y artículos de profesores y profesionales de la Psicología; confiamos en que esta nueva oportunidad que ofrece la Revista Investigación y Pensamiento Crítico sirva de estímulo para publicar y dar a conocer el trabajo profesional y de investigación que están desarrollando los psicólogos. 
Invest. pens. crit.

Vol. 2, No. 3, enero-abril 2014.

pp. 108-117

\section{Referencias}

Difernan, B. (1999). Historia documentada de la Universidad Santa María La Antigua (Vols. I y II). Panamá: Editorial La Antigua, No. 54

Fuentes de Pineda, M. (2013). Programas de postgrado en Ciencias Sociales de la USMA [Presentación de diapositivas]. Reunión de coordinadores de postgrado, Universidad Católica Santa María la Antigua, Panamá

Palacios de Mon, M. (1984). Aspectos históricos de la Asociación Panameña de Psicólogos (APP). Artículo para el Boletín Informativo de la APP.

Palacios de Mon, M. (1990). Escuela de Psicología de la USMA, celebra sus Bodas de Plata. El Panamá América, pp. 42 A

Palacios de Mon, M. (2005). Cuarenta años de fundación de la Escuela de Psicología de la USMA. Panamá: Brochure para la Cena de Gala.

Whittaker, J. (1984). Psicología (4a ed.). México: Editorial Interamericana. 